\title{
SPECTROSCOPIC AND PHOTOMETRIC OBSERVATIONS OF YY ORIONIS
}

\author{
Merle F. Walker \\ Lick Observatory, University of Caljfornia, Santa Cruz \\ Santa Cruz, California, 95064, U. S. A.
}

Three observational studies of YY Ori, the apparently brightest of the YY Ori-type variables in the Orion nebula cluster (Walker 1972), have been carried out. These are:

1. A program of simultaneous spectroscopic and photometric observations. In this program, spectra were obtained on 14 nights at a dispersion of $115 \mathrm{~A} / \mathrm{mm}$ using a Spectracon image converter mounted at the focus of the Bowen $\mathrm{f} / \mathrm{l}$ camera of the coude spectrograph of the 120-inch reflector (Walker 1966, 1969a). The simultaneous photoelectric observations, on the $\underline{U} \underline{B} \underline{V}$ system, were obtained using the 24-inch Lick reflector.

2. In collaboration with $\mathrm{D}$. Burstein, higher dispersion $(0.625 \mathrm{~A} /$ channel) observations were obtained on two nights using the Robinson-Wampler scanner at the Cassegrain focus of the 120 -inch reflector to investigate in greater detail the structure of the emission and inverse $P$ Cyg absorption features in the spectrum.

3. Spectrophotometric observations were obtained using the instrumentation described in (1) above with a wide slit (4" in width) to investigate the form of the energy distribution in the region from 3200A $-5100 \mathrm{~A}$.

These studies have yielded the following results:

1. The characteristic period of the light variation appears to be of the order of several days, with changes of up to one magnitude in B occurring within one day.

2. The amplitude of the light variation is a function of the wavelength, such that $\underline{\Delta B}=1.37 \Delta \underline{V}$ and $\underline{\Delta U}=2.0 \Delta V_{-}$

3. The radial velocities of the Balmer emission lines nearly always increase with increasing quantum number, being highly negative for the lower members of the series and approaching the systemic velocity as the quantum number increases. The velocities of the Ca II and He I emissions agree roughly with the systemic velocity. These results agree with the previous velocity measurements of this star (Walker 1972). Real variations in velocity occur but are not correlated with the spectroscopic appearance or photometric behavior of the system.

4. The intensities of the emission lines of $\mathrm{H}$, He I and $\mathrm{Ca}$ II undergo large variations relative to the continuum. This is illustrated in Figure 1 which reproduces microdensitometer tracings of a selection of the spectra obtained with the Spectracon, arranged in order of decreasing $\mathrm{H}$ emission line intensity. These tracings are on a linear intensity scale, although they have not been corrected 

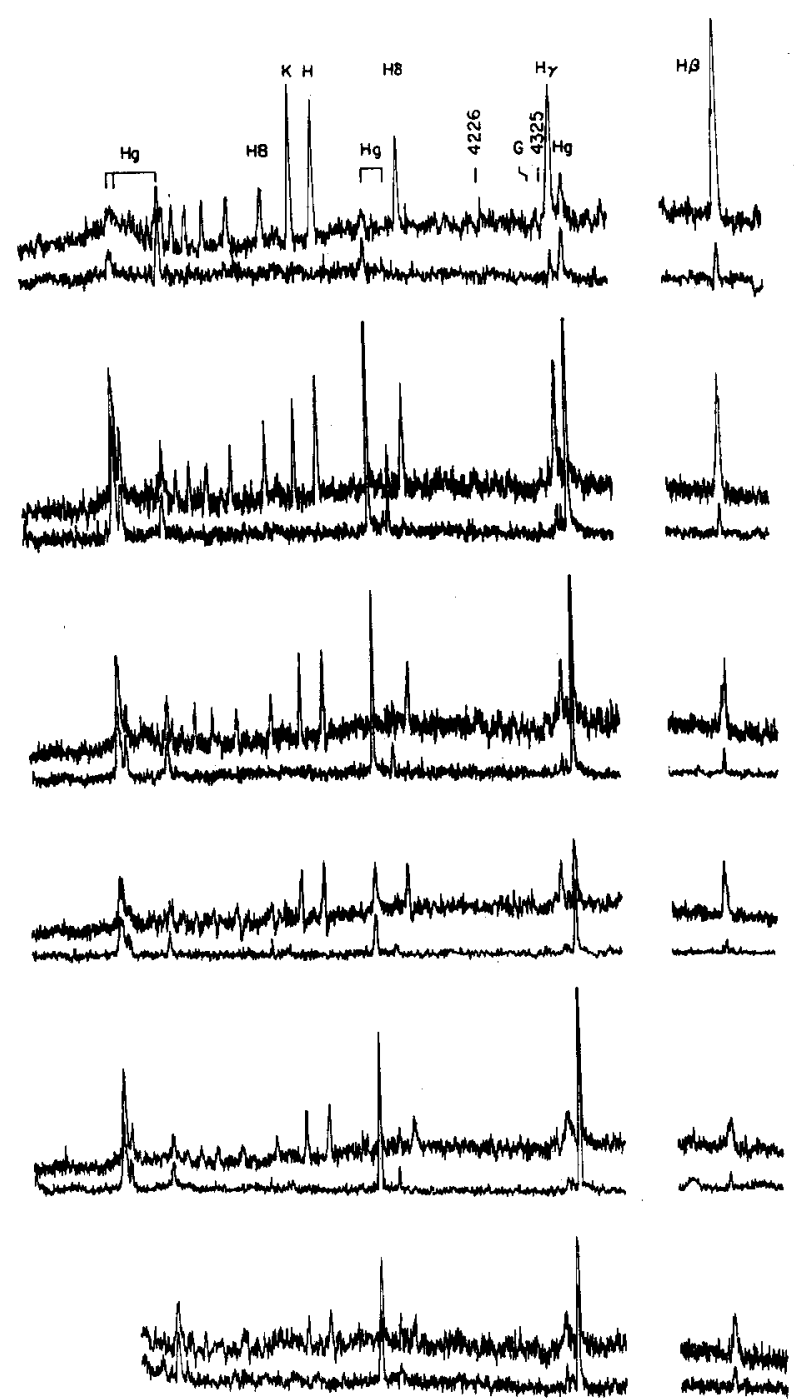

Fig. 1. Microdensitometer tracings of spectra of YY Ori, and the background sky plus nebular spectrum adjacent to the star, arranged in order of decreasing emission line intensity. Top to bottom, UT dates and B magnitudes are: 1971 Jan 20 , $13.4 ; 1972$ Jan 9 , 14.2; 1972 Jan $8,14.6 ; 1971$ oct 1, 13.7; 1972 Jan 6, 14.1; 1971 Nov $25,14.4$. 
for transmission of the atmosphere, telescope and spectrograph or for the sensitivity function of the photocathode of the Spectracon. $H$ and Ca II vary similarly but with variable intensity ratio. He $I$ is more nearly constant and often varies independently. While the emission intensities sometimes follow the light variation, this is not always the case and in general the emission line strengths are not correlated with the brightness of the star.

5. The Balmer emission lines exhibit a complex, variable structure. On five of the 14 nights on which coude spectra were obtained, the $H_{\beta}$ emission was double, the shortward peak having radial velocities of $-155--208 \mathrm{~km} / \mathrm{sec}$ and the longward peak $+130-+213 \mathrm{~km} / \mathrm{sec}$. This emission structure is further $i 11$ ustrated in Figures 2 and 3 which reproduce profiles obtained with the Cassegrain scanner on October 23, 1974 and December 11, 1974 (UT). Figure 2 shows that on October $23, \mathrm{H} \gamma$ was broader than $\mathrm{H} \delta$, being made up of three components, one violet shifted about $-200 \mathrm{~km} / \mathrm{sec}$ from the main peak, and the main peak itself consisting of two components separated by about $90 \mathrm{~km} / \mathrm{sec}$. However, on December 11 (Figure 3) while $\mathrm{H}_{\beta}$ displayed a secondary peak about $+230 \mathrm{~km} / \mathrm{sec}$ redward of the main peak, $\mathrm{H}_{\gamma}$ and $\mathrm{H}_{\delta}$ have widths set by the instrumental profile. The velocities of the longward components of the emission features at $H_{\beta}$ are substantially different from the velocities of the inverse $P$ Cyg absorptions (which average about $+290 \mathrm{~km} / \mathrm{sec}$ ), and thus do not refer to the infatiing gas which produces the inverse $P$ Cyg profiles.

6. The inverse $P$ Cyg absorptions can appear or disappear within intervals at least as short as one day and may be either visible or invisible for several successive days. The occurrance of these features is not correlated with the brightness of the star, unlike SU Ori where such a correlation appears to exist Walker 1969b, 1972). However, the $H$ emission features are noticably asymmetric when strong inverse $P$ Cyg absorptions are present. This is illustrated in Figures 2 and 3 , the observations in Figure 2 having been made when the inverse $P$ Cyg absorptions were strong, and those in Figure 3 when they were very weak.

7. Some changes in the visibility of the late-type photospheric absorption spectrum may occur, but are of the same order as the observational uncertainty. So far as can be determined, no correlation exists between the visibility of the photospheric spectrum and the brightness of the star or the intensity of the emission lines, nor does any detectable change in spectral type occur, although classification of the absorption spectrum is difficult owing to the veiling by the bTue continuum.

8. The energy distribution of the ultraviolet excess and blue continuum, derived from the observed energy curve by subtracting the contribution of the late-type absorption spectrum, is shown in Figure 4. The general appearance of the curve suggests that it results from Balmer and Paschen continuum emission. 
95
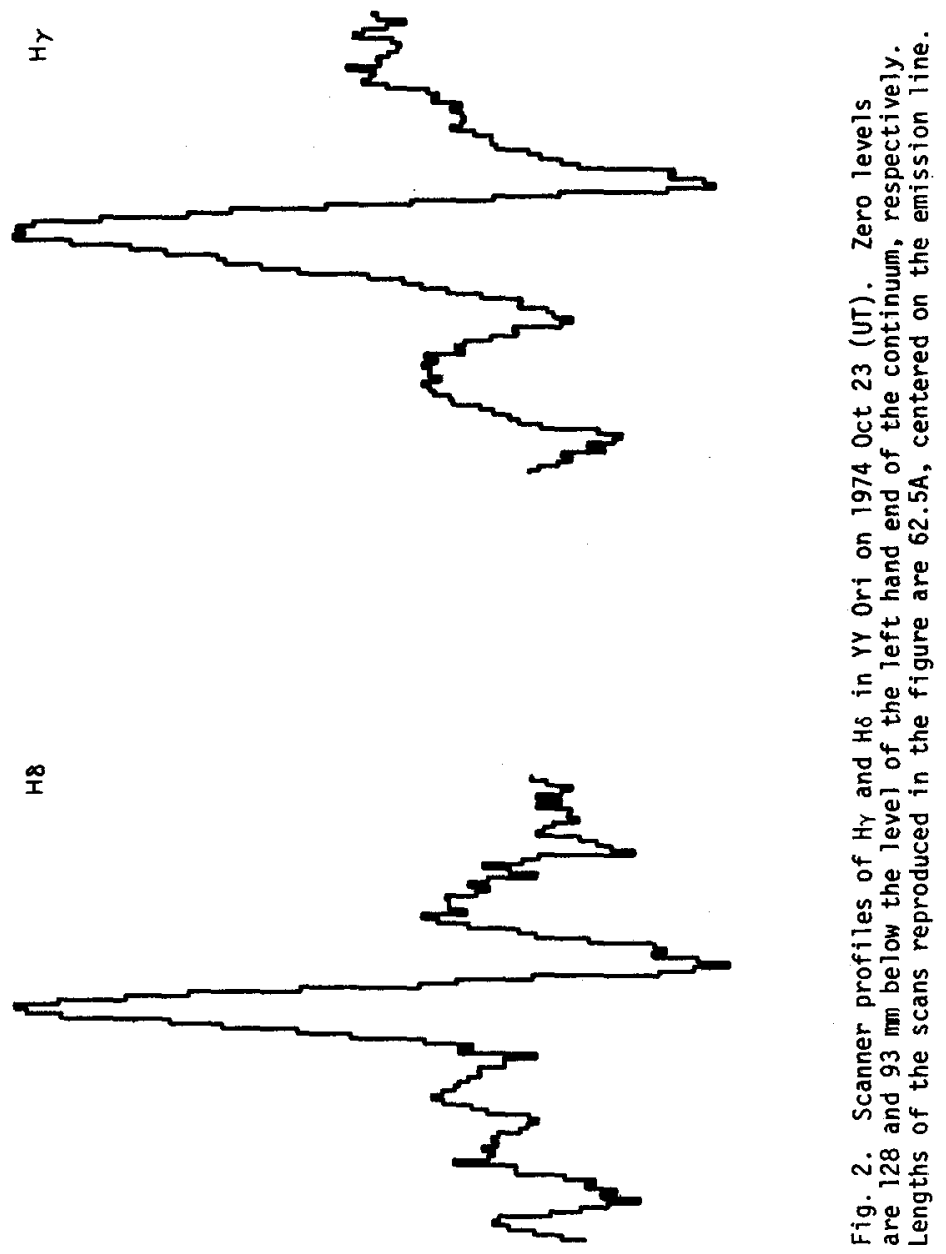

https://doi.org/10.1017/S0252921100069220 Published online by Cambridge University Press 


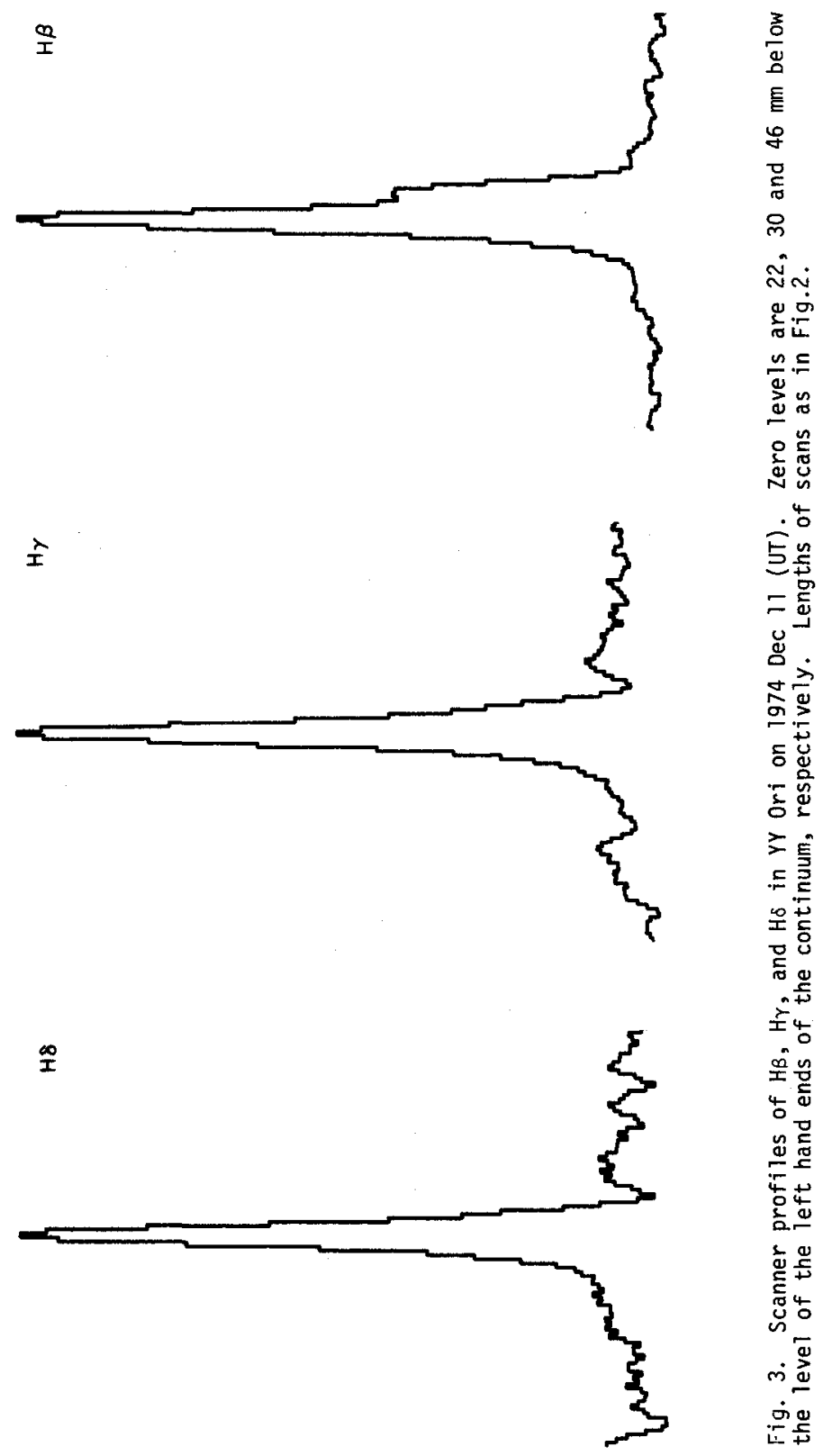


However, while the form of the energy distribution longward of the Balmer limit matches that for Paschen emission of optically thin $H$ at $5000^{\circ} \mathrm{K}$, the intensity ratio on either side of the Balmer limit corresponds to a temperature of about $10,000^{\circ} \mathrm{K}$. Still, improved observational material covering a longer wavelength range will be required before a definitive comparison with theory can be made.

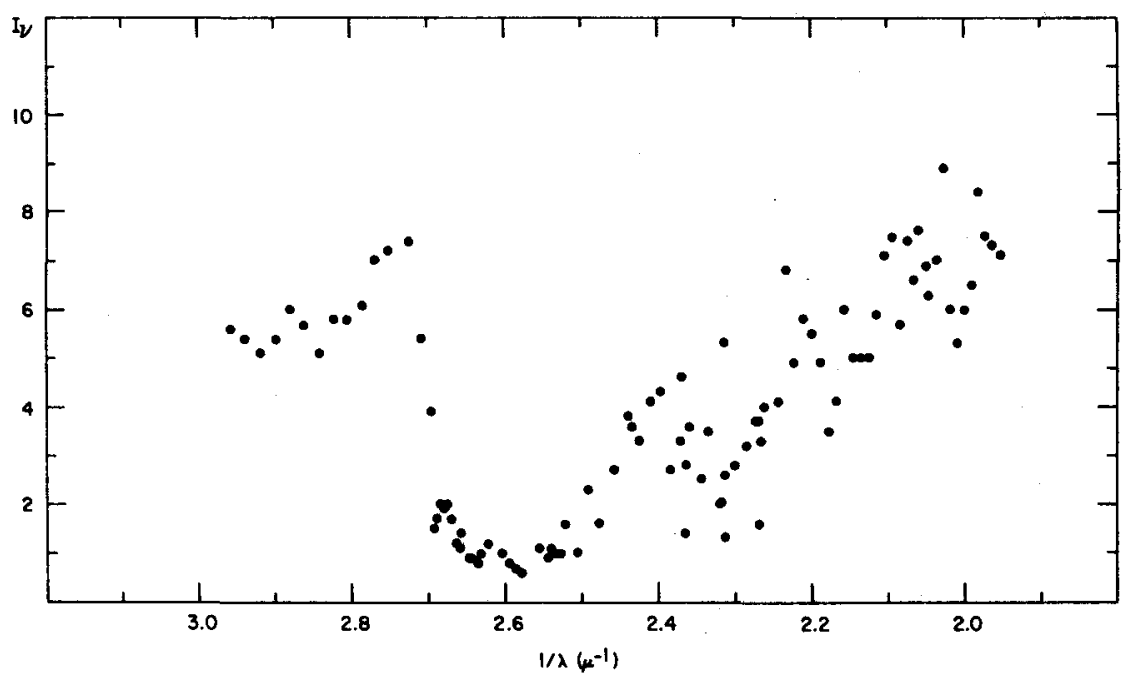

Fig. 4. Energy distribution of the ultraviolet excess and blue continuum in $Y Y$ Ori on 1971 Jan 20 (UT).

These observational results lead to the following conclusions:

1. The light variations of $Y Y$ Ori probabiy result mainiy from variable obscuration by remnants of the pre-stellar cloud. The observed wavelength dependence of the amplitude of the light variation is consistent with either variability due to changes in temperature or to variable absorption in the line of sight. Since no spectral type variation is observed, the wavelength dependence of the light variation, together with the time scale of the light variations and the lack of correlation between these variations and either the emission line intensity or visibility of the photospheric absorption spectrum suggests that the light variations result primarily from variable obscuration. However, this conclusion must be checked by obtaining additional spectra of higher quality.

2. The region and material producing the emission spectrum is different from that causing the light variations, and the region in which the $\mathrm{H}$ emission is produced is different from those in which the He I or Ca II emissions originate. 
3. The spatial and physical structure of the $\mathrm{H}$ emission region itself is complex and variable.

4. The infalling gas which produces the inverse $P$ Cyg absorptions is different from either the material producing the variable absorption or the emission spectrum.

5. The ultraviolet excess and biue continuum are probably the result of Balmer and Paschen continuum emission, although the agreement of the observed energy distribution with theory is not too satisfactory.

The present studies thus demonstrate that the structure of YY Ori is extremely complex and show that extensive observational material must be obtained before a definitive model of this sytem can be constructed. Moreover, the lack of variation of the $H$ emission line velocity with quantum number found in other YY Ori variables (Walker 1972) and the apparent correlation of inverse $P$ Cyg absorption line strength with brightness in SU Ori (Walker 1969, 1972) indicate that the same model may not apply to all YY Ori stars, so that simultaneous spectroscopic and photometric observations of a number of these systems should be obtained. It is clear, however, that in the end such studies will add enormously to our understanding of the last stages of the process of stellar formation.

\section{References:}

Walker, M. F. 1966, Adv. in Electronics, 22B, 761. Walker, M. F. 1969a, Adv. in Electronics, 28B, 773.

Waiker, M. F. 1969b, Non-periodic Phenomena in Variable Stars, ed. L. Detre (Budapest: Academic Press), p. 103.

Walker, M. F. 1972, Ap. J., 175, 89.

\section{I S CUSS I O N OF paper by WALKER:}

APPENZELLER: In your 1972 paper on the YY orionis stars you describe a correlation between the brightness and the strength of the red shifted absorption component of the Balmer lines for the star SU Orionis. In the case of YY Orionis, you did not find such a correlation. Do you suggest that there is a physical difference between these two objects?

WALKER: It is quite possible, although the number of observations of SU Ori is very small and the apparent correlation may be accidental; a more extensive study of su ori should be made. The observations of SCrA, reported by Mundt, appear to be more extensive and, at the moment, this material may provide more conclusive evidence for the reality of such a correlation for some YY Ori stars, but $1 t$ is clear that more extended observations of a larger number of $Y Y$ Ori stars are required to give a definite answer. 
THE: In the $V, B-V$ diagram you showed the size of the direction coefficient of the line giving the correlation of the changes of $\mathrm{V}$ and those of $\mathrm{B}-\mathrm{V}$.

WALKER: The lines in the figures correspond to: $\Delta B=1.37 \Delta \mathrm{V}$ and $\Delta U=2.00 \Delta \mathrm{V}$. In view of the size of the observational error (due to the faintness of the star and the irregular nebular background against which it must be observed,) these relations do not depart significantly from the relation to be expected if the light variations are due to variabie opacity by "normal" interstellar material: $\Delta \mathrm{B}=1.33 \Delta \mathrm{V}$ and $\Delta \mathrm{U}=1.58 \Delta \mathrm{V}$.

MIRZOYAN: If I understood correctly, in the spectrum of YY or 1 we observe in different periods inverse normal or no $P$ Cygni profiles at all. Is that correct?

WALKER: No, normal $P$ Cygni profiles have never been observed, only inverse $P$ Cygni absorption or no absorption. 